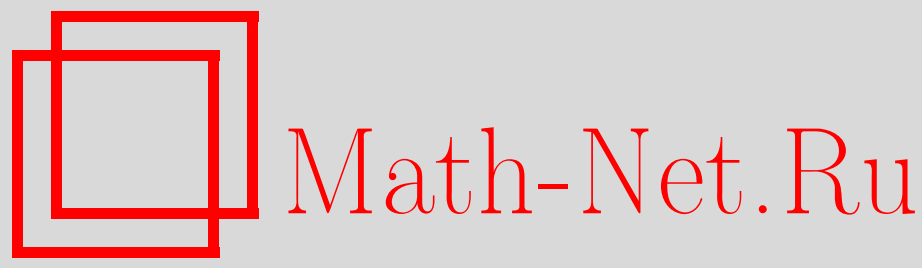

О. В. Капцов, Локальный алгебраический анализ дифференциальных систем, ТМФ, 2015, том 183, номep 3, 342-358

DOI: https://doi.org/10.4213/tmf8760

Использование Общероссийского математического портала Math-Net.Ru подразумевает, что вы прочитали и согласны с пользовательским соглашением http://www.mathnet.ru/rus/agreement

Параметры загрузки:

IP : 34.227 .88 .159

26 апреля 2023 г., 11:31:36

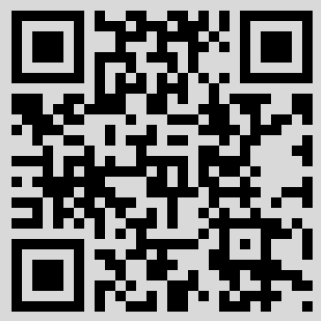




\title{
ЛОКАЛЬНЫЙ АЛГЕБРАИЧЕСКИЙ АНАЛИЗ ДИФФЕРЕНЦИАЛЬНЫХ СИСТЕМ
}

\begin{abstract}
Предложен новый подход к исследованию совместности уравнений с частными производными. Этот подход является синтезом метода Рикье, теории базисов Грёбнера и элементов алгебраической геометрии. В качестве приложений рассмотрены системы, включающие волновое уравнение и уравнение синусгордон.
\end{abstract}

Ключевые слова: совместность дифференциальных уравнений, редукция, базис Грёбнера.

DOI: $10.4213 / \operatorname{tmf} 8760$

\section{1. ВВЕДЕНИЕ}

Проблема совместности уравнений с частными производными начала активно изучаться на рубеже девятнадцатого и двадцатого веков в работах Кёнига, Рикье, Жане, Картана и других авторов. Обзор работ этого времени представлен в монографиях [1], [2]. После некоторого периода затишья интерес к этому вопросу возродился в связи с возникающими приложениями и появлением новых задач. На этом этапе в теории дифференциальных уравнений стал использоваться язык расслоений, гомологической и коммутативной алгебры [3]-[6]. В последнее время основные акценты в исследованиях сместились в сторону алгоритмических и вычислительных вопросов [7]-[9]. Это объясняется успехами в развитии компьютерной алгебры. Наибольший прогресс был достигнут в исследовании совместности систем алгебраических уравнений благодаря теории базисов Грёбнера [10], [11]. В приложениях переопределенные системы возникают в связи с исследованием симметрий и законов сохранения уравнений математической физики [12], [13]. Дифференциальные связи успешно применялись для построения решений уравнений газовой динамики [14] и квантовой механики [15]. Метод обратной задачи [16] использует условия совместности линейных систем уравнений с частными производными.

В настоящей работе предложен алгебро-аналитический подход к изучению некоторых локальных свойств уравнений с частными производными. В данном подходе

* Институт вычислительного моделирования СО РАН, Красноярск, Россия.

E-mail: kaptsov@icm.krasn.ru

${ }^{\dagger}$ Сибирский федеральный университет, Красноярск, Россия 
используются идеи алгебраической геометрии и базисов Грёбнера. Алгебраическая структура, в рамках которой ведутся построения, состоит из локального дифференциального кольца, отношений на кольце и полугруппы, действующей на кольце.

В разделе 2 вводится бесконечномерное пространство - аналог пространства бесконечных джетов. Каждой точке $a$ этого пространства сопоставляется локальная дифференциальная алгебра $\mathcal{F}(a)$ сходящихся рядов. Каждое подмножество $S \subset \mathcal{F}(a)$ порождает дифференциальный идеал, и основным объектом исследования становятся пассивные множества конечно порожденных дифференциальных идеалов алгебр $\mathcal{F}(a)$. Пассивное множество является подобием базиса Грёбнера идеала кольца полиномов, однако наше определение пассивности не использует порядка на кольце. В конце раздела 2 получено необходимое условие пассивности множества.

В разделе 3 доказываются утверждения, аналогичные тем, что известны в теории базисов Грёбнера. В отличие от стандартного подхода, применяемого к алгебре полиномов, мы используем разбиение алгебры $\mathcal{F}(a)$, действие полугруппы, согласованное с разбиением, и теорему Вейерштрасса о делении степенных рядов. Дается определение редукции ряда относительно некоторых подмножеств алгебры $\mathcal{F}(a)$, нормальной формы ряда, $\tau$-ряда (аналога $S$-многочлена), канонического множества дифференциального идеала. Описывается способ приведения сходящегося дифференциального ряда к нормальной форме. В терминах $\tau$-рядов получен удобный критерий пассивности. Доказано, что если дифференциальный идеал обладает пассивным множеством, то существует единственное каноническое множество, порождающее данный идеал.

В заключительном разделе 4 описывается схема построения пассивного множества заданного дифференциального идеала, которая опирается на некоторое уточнение теоремы о неявных функциях, доказанное в работе. Хотя эта схема не является универсальной, она может оказаться полезной при исследовании совместности конкретных систем уравнений с частными производными. В качестве приложения данной схемы рассматриваются два примера. В первом примере исследуется совместность системы, состоящей из уравнения синус-гордон и уравнения третьего порядка, заданного высшей симметрией. Во втором примере строятся пассивные множества, связанные с трехмерным волновым уравнением для звука в неоднородной среде. Указывается, как использовать пассивные множества при построении решений уравнений.

\section{2. ПАССИВНЫЕ МНОЖЕСТВА}

Пусть $\mathbb{N}$ обозначает множество неотрицательных целых чисел, $\mathbb{N}_{m}=\{1, \ldots, m\}$. Тогда $\mathbb{N}^{n}$ - полугруппа, порожденная образующими

$$
e_{1}=(1,0, \ldots, 0), \quad \ldots, \quad e_{n}=(0, \ldots, 0,1) .
$$

Пусть $\mathbb{K}$ - поле, полное относительного некоторой нормы. Обозначим через $\mathbb{K}^{\infty}$ множество семейств $\left\{a_{\alpha}^{i}\right\}_{\alpha \in \mathbb{N}^{n}}^{i \in \mathbb{N}_{m}}, a_{\alpha}^{i} \in \mathbb{K}$. Операции сложения семейств

$$
\left\{a_{\alpha}^{i}\right\}+\left\{b_{\alpha}^{i}\right\}=\left\{a_{\alpha}^{i}+b_{\alpha}^{i}\right\}
$$

и умножения на элементы поля

$$
c\left\{a_{\alpha}^{i}\right\}=\left\{c a_{\alpha}^{i}\right\}
$$


задают структуру векторного пространства на $\mathbb{K}^{\infty}$. Пространство $\mathbb{K}^{n} \times \mathbb{K}^{\infty}$ обозначается как $\mathbb{K}^{n+\infty}$. Часто подобные пространства называются пространствами джетов, или продолженными пространствами.

Каждой точке $a \in \mathbb{K}^{n+\infty}$ сопоставим некоторую $\mathbb{K}$-алгебру сходящихся степенных рядов следующим образом. Если $L-$ конечномерное координатное подпространство в $\mathbb{K}^{n+\infty}$, то $\mathbb{K}^{n+\infty}$ представляется в виде прямой суммы $L \oplus M$, где $M-$ прямое дополнение к $L$. Проектор из $\mathbb{K}^{n+\infty}$ на $L$ вдоль $M$ обозначим через $\pi_{L}$. C помощью координатного изоморфизма $\phi$ пространство $L$ отображается на $\mathbb{K}^{s}, s=\operatorname{dim} L$. Точке $a \in \mathbb{K}^{n+\infty}$ сопоставим точку $a_{L}=\phi\left(\pi_{L}(a)\right) \in \mathbb{K}^{s}$. Множество сходящихся степенных рядов с центром в точке $a_{L}$, зависящих от конечного числа переменных, обозначим через $\mathcal{F}\left(a_{L}\right)$.

Если подпространство $L$ содержится в подпространстве $L^{\prime}$, то мы считаем, что кольцо $\mathcal{F}\left(a_{L}\right)$ канонически вложено в $\mathcal{F}\left(a_{L^{\prime}}\right)$. Тогда объединение

$$
\mathcal{F}(a)=\bigcup_{L \in \mathbb{K}^{n+\infty}} \mathcal{F}\left(a_{L}\right)
$$

является $\mathbb{K}$-алгеброй, соответствующей точке $a$. Декартовы координаты на $\mathbb{K}^{n+\infty}$ обозначаются как $x_{1}, \ldots, x_{n}, \ldots, u_{\alpha}^{i}, \ldots$, a все множество декартовых координат обозначается через $Y$. Оно разбивается на два подмножества

$$
X=\left\{x_{1}, \ldots, x_{n}\right\}, \quad U=\left\{u_{\alpha}^{i}: i \in \mathbb{N}_{m}, \alpha \in \mathbb{N}^{n}\right\} .
$$

Согласно монографии [17] алгебра $\mathcal{F}(a)$ является локальной с максимальным идеалом

$$
\mathfrak{M}(a)=\{f \in \mathcal{F}(a): f(a)=0\} .
$$

На $\mathcal{F}(a)$ действуют $n$ операторов дифференцирования

$$
D_{i} f=\frac{\partial f}{\partial x_{i}}+\sum_{\substack{j \in \mathbb{N}_{m} \\ \alpha \in \mathbb{N}^{n}}} \frac{\partial f}{\partial u_{\alpha}^{j}} u_{\alpha+e_{i}}^{j} .
$$

Следовательно, $\mathcal{F}(a)$ - дифференциальная алгебра. Элементы алгебры $\mathcal{F}(a)$ называются сходящимися дифференииальными рядами.

Полезно также рассматривать дизъюнктное объединение алгебр (“пучок" локальных алгебр над $\mathbb{K}^{n+\infty}$ )

$$
\mathcal{F}=\bigcup_{a \in \mathbb{K}^{n+\infty}} \mathcal{F}(a)
$$

ОПРЕДЕЛЕНИЕ 1. Локальной аналитической дифференциальной системой называется упорядоченная тройка множеств $(\mathbb{K}, Y, S)$, где $S$ - подмножество в $\mathcal{F}$.

Локальные аналитические дифференциальные системы можно рассматривать как алгебраическую формализацию уравнений с частными производными.

Дифференциальный идеал в $\mathcal{F}(a)$, порожденный множеством $S$, будет обозначаться как $\langle\langle S\rangle\rangle$, произведение операторов $D_{1}^{\alpha_{1}} \ldots D_{n}^{\alpha_{n}}$ - как $D^{\alpha}$; пусть iv $(f)$ - множество переменных, от которых зависит ряд $f \in \mathcal{F}$. Если $Y^{\prime}-$ непустое подмножество в $Y$, тогда

$$
\mathbb{K}\left[\left\langle Y^{\prime}\right\rangle\right]_{a}=\left\{f \in \mathcal{F}(a): \operatorname{iv}(f) \subseteq Y^{\prime}\right\}
$$

является подалгеброй алгебры $\mathcal{F}(a)$. 
ОПРЕДЕЛЕНИЕ 2. Орбитой множества $S \subset \mathcal{F}$ называется множество

$$
O(S)=\left\{D^{\alpha} s: \alpha \in \mathbb{N}^{n}, s \in S\right\}
$$

Используя аналогию с алгебраической геометрией и теорией локальных аналитических алгебр [18], введем следующие понятия.

ОПРЕДЕЛЕНИЕ 3 . Пусть $S \subset \mathcal{F}(a)$, где $a$ - такая точка в $\mathbb{K}^{n+\infty}$, что орбита множества $S$ содержится в максимальном идеале $\mathfrak{M}(a)(2.3)$. Тогда фактор-алгебра

$$
\mathcal{O}_{S}(a)=\mathcal{F}(a) /\langle\langle S\rangle\rangle
$$

называется локалъной алгеброй точки а. Если $\mathcal{O}_{S}(a)$ изоморфна некоторой алгебре $\mathbb{K}\left[\left\langle Y^{\prime}\right\rangle\right]_{a}$, то а называется неособой точкой для $S$.

Далее мы получим условия того, что точка является неособой. В случае локальных аналитических алгебр такие условия следуют из теоремы Якоби о неявных функциях [18].

ОПРЕДЕЛЕНИЕ 4. Множество $\mathcal{B} \subset \mathcal{F}(a)$ называется нормализованным, если выполнены следующие условия:

1) любой элемент $f \in \mathcal{B}$ имеет вид

$$
f=u_{\alpha}^{i}+g
$$

при этом переменные $u_{\alpha}^{i}$ образуют подмножество $\mathcal{L}$ в $U, \operatorname{aiv}(g) \in Y \backslash \mathcal{L}$;

2) если $f_{1}=u_{\alpha}^{i}+g_{1}, f_{2}=u_{\alpha}^{i}+g_{2} \in \mathcal{B}$, то $g_{1}=g_{2}$.

В этом случае $\mathcal{L}$ называется множеством главных переменных, а $\mathcal{P}=Y \backslash \mathcal{L}$ называется множеством параметрических переменных системы $\mathcal{B}$.

ЗАмЕчАниЕ 1. В теории Жане-Рикье [19] используются понятия главных и параметрических производных ортономных систем.

ОПРЕДЕЛЕНиЕ 5. Система образующих $\mathcal{B}$ идеала алгебры $\mathcal{F}(a)$ называется нормализованной, если $\mathcal{B}$ - нормализованное множество в $\mathcal{F}(a)$.

Всюду в дальнейшем мы будем использовать понятие системы образующих идеала в стандартном смысле [17], несмотря на то что рассматриваемые идеалы часто будут дифференциальными.

ЗАмЕчАниЕ 2. Для заданного дифференциального идеала нормализованная система образующих может быть не единственной. Достаточные условия существования нормализованной системы образующих получены в статье [20].

УтВеРЖДЕНИЕ 1. Точка $a \in \mathbb{K}^{n+\infty}$ является неособой для $S \subset \mathcal{F}(a)$, если идеал $\langle\langle S\rangle\rangle$ обладает нормализованной системой образующих $\mathcal{B} u\langle\langle S\rangle\rangle \mathcal{F}(a)$. При этом локальная алгебра $\mathcal{O}_{S}(a)$ точки а изоморфна алгебре $\mathbb{K}[\langle\mathcal{P}\rangle]_{a}$, где $\mathcal{P}$ - множество параметрических переменных системы $\mathcal{B}$.

ДокАЗАтЕльство. Возьмем произвольный ряд $f \in \mathcal{F}(a)$. Если $f$ не зависит от главных переменных нормализованной системы образующих $\mathcal{B}$ идеала $\langle\langle S\rangle\rangle$, то $f \in \mathbb{K}[\langle\mathcal{P}\rangle]_{a}$. 
Предположим, что $f$ зависит от главных переменных $y_{1}, \ldots, y_{p}$ системы образующих $\mathcal{B}$. Тогда найдутся ряды $f_{1}, \ldots, f_{p} \in \mathcal{B}$ такие, что

$$
f_{i}=y_{i}+h_{i}, \quad i=1, \ldots, p, \quad h_{i} \in \mathbb{K}[\langle\mathcal{P}\rangle]_{a} .
$$

Используя теорему Вейерштрасса о делении [18], [20], [21], мы можем представить ряд $f$ в виде

$$
f=\sum_{i=1}^{p} q_{i} f_{i}+r, \quad r, q_{i} \in \mathcal{F}(a),
$$

где $r$ не зависит от главных переменных из $\mathcal{L}$ и определяется однозначно. Следовательно, $r$ лежит в $\mathbb{K}[\langle\mathcal{P}\rangle]_{a}$.

ОПРЕДЕЛЕНиЕ 6. Сходящийся дифференциальный ряд $f \in \mathcal{F}$ вида (2.6) называется разрешимым относительно $u_{\alpha}^{i}$, если $g$ не зависит от элементов орбиты $O\left(u_{\alpha}^{i}\right)$.

ОПРЕДЕЛЕНИЕ 7. Множество $S \subset \mathcal{F}(a)$ называется пассивным (в точке $a$ ), если:

1) существует нормализованная система образующих $\mathcal{B}$ дифференциального идеала $\langle\langle S\rangle\rangle$;

2) каждый ряд $f \in S$ разрешим относительно некоторого элемента $u_{\alpha}^{i}$ и при этом объединение орбит элементов $u_{\alpha}^{i}$ совпадает с множеством главных переменных системы $\mathcal{B}$.

В этом случае объединение орбит элементов $u_{\alpha}^{i}$ обозначается как $O(\operatorname{st} S)$, а элемент, относительно которого разрешим ряд $f \in S$, обозначается через st $f$.

Ниже приведено необходимое условие пассивности множества.

ЛЕмма 1. Если $S$ - пассивное множество, то любой элемент идеала $\langle\langle S\rangle\rangle$ зависит хотя бъ от одного элемента орбиты $O(\operatorname{st} S)$.

ДокАзАтЕльство. Предположим, что найдется ненулевой ряд $h \in\langle\langle S\rangle\rangle$, не зависящий от элементов орбиты $O(\operatorname{st} S)$. Так как $S$ - пассивное множество и ряд $h$ лежит в идеале, порожденном нормализованной системой образующих $\mathcal{B}$, мы имеем

$$
h=\sum_{j=1}^{p} a_{j} b_{j}
$$

где $a_{j} \in \mathcal{F}(a), b_{j} \in \mathcal{B}$. Поскольку $b_{1}, \ldots, b_{p}$ принадлежат нормализованному множеству $\mathcal{B}$, матрица Якоби от этих рядов по переменным st $b_{1}, \ldots$, st $b_{p}$ является единичной.

Теперь обозначим переменные, от которых зависят ряды $a_{1}, \ldots, a_{p}, b_{1}, \ldots, b_{p}$, через $y_{1}, \ldots, y_{r}$, полагая при этом $y_{1}=$ st $b_{1}, \ldots, y_{p}=$ st $b_{p}$. Выполним замену переменных

$$
z_{1}=b_{1}, \quad \ldots, \quad z_{p}=b_{p} .
$$

По теореме о неявных функциях последние соотношения можно разрешить относительно $y_{1}, \ldots, y_{p}$. Подставляя найденные $y_{1}, \ldots, y_{p}$ в $(2.7)$, получаем представление

$$
h=\sum_{j=1}^{p} \tilde{a}_{j} z_{j}
$$


где $h$ может зависеть только от $y_{p+1}, \ldots, y_{r}$. Поскольку правая часть последнего соотношения зависит хотя бы от одной из переменных $z_{1}, \ldots, z_{p}$, мы приходим к противоречию.

Хотелось бы обратить внимание на следующий вопрос: верно ли утверждение, что если $a$ - неособая точка для $S \subset \mathcal{F}(a)$, то идеал $\langle\langle S\rangle\rangle$ обладает пассивным множеством? Положительный ответ на этот вопрос означал бы, что пассивность равносильна регулярности.

ЗАмЕчАниЕ 3. Пассивное множество является аналогом базиса Грёбнера идеала кольца многочленов. Однако в нашем определении не используется порядок. С другой стороны, порядок и другие алгебраические структуры полезны для получения критерия пассивности множества.

\section{3. СТРАТИФИЦИРОВАННЫЕ МНОЖКСТВА И РЕДУКЦИИ}

Введем сначала определения, которые нам скоро понадобятся. В дальнейшем $(\Gamma, \leqslant)$ обозначает вполне упорядоченное множество. Пусть на множестве $A$ действует полугруппа $G$, т. е. задано отображение $(g, a) \rightarrow g a$ множества $G \times A$ в $A$, удовлетворяющее условию

$$
g_{1}\left(g_{2} a\right)=\left(g_{1} g_{2}\right) a \quad \text { для всех } a \in A, \quad g_{1}, g_{2} \in G .
$$

ОПРЕДЕЛЕНиЕ 8. Пусть $\left\{A_{\gamma}\right\}_{\gamma \in \Gamma}$ - разбиение множества $A$. Полугруппа $G$, действующая на $A$, сохраняет разбиение, если для любого $g \in G$, для любого $\gamma \in \Gamma$ и для всех $a, b \in A_{\gamma}$ найдется $\gamma^{\prime}$ такое, что $g a, g b \in A_{\gamma^{\prime}}$.

ОПредЕЛЕНИЕ 9. Пусть задано разбиение $\left\{A_{\gamma}\right\}_{\gamma \in \Gamma}$ множества $A$, полугруппа $G$, действующая на $A$ и сохраняющая это разбиение. Множество $A$ называется $c m p a m u-$ фицированным $G$-множеством, если на нем задано рефлексивное (иррефлексивное) транзитивное отношение $\prec$, для всех $g \in G$ удовлетворяющее условиям:

1) $a \prec b$ влечет $g a \prec g b$;

2) $a \prec g a$ для любого $a \in A$.

Введем множества

$$
\widehat{\mathcal{F}}(a)=\mathcal{F}(a) \backslash \mathbb{K}[\langle X\rangle]_{a}, \quad \widehat{\mathcal{F}}=\bigcup_{a \in \mathbb{K}^{n+\infty}} \widehat{\mathcal{F}}(a),
$$

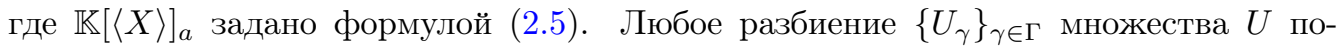
рождает разбиения множеств $\widehat{\mathcal{F}}(a), \widehat{\mathcal{F}}$ следующим образом. Рассмотрим семейство множеств

$$
Y_{\gamma}=X \cup\left(\bigcup_{\gamma^{\prime} \leqslant \gamma} U_{\gamma^{\prime}}\right)
$$

Возьмем точку $a \in \mathbb{K}^{n+\infty}$ и подалгебру

$$
\mathcal{F}_{\gamma}(a)=\left\{f \in \mathcal{F}(a): \operatorname{iv}(f) \in Y_{\gamma}\right\}
$$

Тогда семейства $\left\{\mathcal{F}_{\gamma}(a)\right\}_{\gamma \in \Gamma},\left\{\mathcal{F}_{\gamma}\right\}_{\gamma \in \Gamma}$, где

$$
\mathcal{F}_{\gamma}=\bigcup_{a \in \mathbb{K}^{n+\infty}} \mathcal{F}_{\gamma}(a)
$$


порождают разбиения $\left\{\Phi_{\gamma}(a)\right\}_{\gamma \in \Gamma},\left\{\Phi_{\gamma}\right\}_{\gamma \in \Gamma}$ множеств $\widehat{\mathcal{F}}(a), \widehat{\mathcal{F}}$ на блоки:

$$
\Phi_{\gamma}(a)=\mathcal{F}_{\gamma}(a) \backslash\left(\bigcup_{\gamma^{\prime}<\gamma} \mathcal{F}_{\gamma^{\prime}}(a)\right), \quad \Phi_{\gamma}=\mathcal{F}_{\gamma} \backslash\left(\bigcup_{\gamma^{\prime}<\gamma} \mathcal{F}_{\gamma^{\prime}}\right) .
$$

Разбиение $\left\{\Phi_{\gamma}\right\}_{\gamma \in \Gamma}$ индуцирует иррефлексивное транзитивное отношение на $\widehat{\mathcal{F}}$

$$
f \prec g \Longleftrightarrow \exists \gamma, \gamma^{\prime}: f \in \Phi_{\gamma}, g \in \Phi_{\gamma^{\prime}}, \gamma<\gamma^{\prime}
$$

и рефлексивное транзитивное отношение

$$
f \preceq g \quad \Longleftrightarrow \quad \exists \gamma, \gamma^{\prime}: f \in \Phi_{\gamma}, g \in \Phi_{\gamma^{\prime}}, \gamma \leqslant \gamma^{\prime} .
$$

Аналогичным образом вводятся отношения $\prec$ и $\preceq$ на $\widehat{\mathcal{F}}(a)$.

На множествах $U, \widehat{\mathcal{F}}(a), \widehat{\mathcal{F}}$ действие полугруппы $\mathbb{N}_{-0}^{n}=\mathbb{N}^{n} \backslash \overrightarrow{0}$, где $\overrightarrow{0}$ - кортеж из нулей, задается формулами

$$
\alpha u_{\beta}^{i}=u_{\alpha+\beta}^{i}, \quad \alpha f=D^{\alpha}(f) .
$$

Всюду в дальнейшем предполагается, что $\widehat{\mathcal{F}}$ и $\widehat{\mathcal{F}}(a)$ являются стратифицированными $\mathbb{N}_{-0}^{n}$-множествами с отношением (3.2). В работе [20] показано, что если $U$ является стратифицированным $\mathbb{N}_{-0}^{n}$-множеством, то $\widehat{\mathcal{F}}$ - также стратифицированное $\mathbb{N}_{-0}^{n}$-множество.

ОПРЕДЕЛЕНИЕ 10. Подмножество $S$ в $\mathcal{F}$ называется слабо разрешимым, если каждый ряд $f \in S$ имеет вид $f=u_{\alpha}^{i}+h$ с некоторыми $u_{\alpha}^{i} \in U, h \in \mathcal{F}$ такими, что $h \prec u_{\alpha}^{i}$. При этом элемент $u_{\alpha}^{i}$ называется старшим членом $f$ и обозначается KaK lt $f$.

Следующее утверждение позволяет ввести “частичное деление" в алгебре $\mathcal{F}(a)$.

УтВЕРЖДЕНИЕ 2. Пусть $f-$ произволъный ряд из алгебры $\mathcal{F}(a)$ u $u_{\alpha}^{i} \in \operatorname{iv}(f)$. Предположим, что ряд $g \in \mathcal{F}(a)$ имеет вид $g=u_{\beta}^{i}+h$, где $u_{\beta}^{i} \in U, h \prec u_{\beta}^{i}$, u найдется элемент $\delta \in \mathbb{N}^{n}$ такой, что $u_{\alpha}^{i}=u_{\beta+\delta}^{i}$. Тогда существуют единственные ряды $q, r \in \mathcal{F}(a)$, удовлетворяющие соотношениям

$$
\begin{gathered}
f=q D^{\delta} g+r, \\
q \preceq f, \quad r \preceq f, \quad u_{\alpha}^{i} \notin \operatorname{iv}(r) .
\end{gathered}
$$

ДокАзАтЕльство. Из теоремы Вейерштрасса о делении [18], [21] следует существование и единственность рядов $q, r \in \mathcal{F}(a)$, удовлетворяющих (3.5) и соотношениям

$$
\operatorname{iv}(q) \subseteq\left(\operatorname{iv}(f) \cup \operatorname{iv}\left(D^{\delta} g\right)\right), \quad \operatorname{iv}(r) \subset\left(\operatorname{iv}(f) \cup \operatorname{iv}\left(D^{\delta} g\right)\right), \quad u_{\alpha}^{i} \notin \operatorname{iv}(r) .
$$

Поскольку $\operatorname{lt}\left(D^{\delta} g\right)=u_{\alpha}^{i} \in \operatorname{iv}(f)$, мы имеем

$$
D^{\delta} g \preceq f .
$$

Тогда из формул (3.7) и (3.8) следует (3.6). 
Будем говорить, что $f \in \mathcal{F}(a)$ одношагово редуиируется к ряду $r \in \mathcal{F}(a)$ относительно $g$, если выполнены условия утверждения 2. Одношаговая редукция обозначается как $f \underset{g}{\rightarrow} r$.

ОПРЕДЕЛЕНИЕ 11. Пусть $f \in \mathcal{F}(a)$, а $S=\left\{f_{1}, \ldots, f_{k}\right\} \subset \mathcal{F}(a)$ - слабо разрешимое подмножество. Ряд $f$ редуиируется $к$ ряду $r \in \mathcal{F}(a)$ относительно $S$, если существует конечная последовательность одношаговых редукций вида

$$
f \underset{f_{i_{1}}}{\longrightarrow} r_{1} \underset{f_{i_{2}}}{\longrightarrow} r_{2} \underset{f_{i_{3}}}{\longrightarrow} \cdots \underset{f_{i_{p}}}{\longrightarrow} r
$$

где $f_{i_{j}} \in S$. Эта последовательность редукций кратко обозначается как $f \underset{S}{\longrightarrow} r$.

ОПРЕДЕЛЕНИЕ 12. Будем говорить, что ряд $f \in \mathcal{F}(a)$ не редуиируемый относительно слабо разрешимого множества $S$, если $\operatorname{iv}(f) \cap O(\operatorname{lt} S)=\varnothing$, где

$$
\text { lt } S=\{\text { lt } f: f \in S\} .
$$

ОПРЕДЕЛЕНИЕ 13. Ряд $r \in \mathcal{F}(a)$ называется нормалъной формой ряда $f \in \mathcal{F}(a)$ относительно слабо разрешимого подмножества $S \subset \mathcal{F}(a)$, если $f \underset{S}{\longrightarrow} r$ и $r$ - ряд, не редуцируемый относительно $S$.

Нормальная форма ряда $f$ относительно $S$ обозначается через $\mathrm{NF}(f \downarrow S)$.

ЛЕмма 2. Пусть $S$ - слабо разрешимое подмножество в $\mathcal{F}(a)$. Тогда ряд $f \in \mathcal{F}(a)$ либо редуцируется $к$ нормальной форме $\mathrm{NF}(f \downarrow S)$ относительно $S$, либо является не редуцируемым относительно $S$.

ДокАзАтЕльство. Если $\operatorname{iv}(f) \cap O(\operatorname{lt} S)=\varnothing$, то $f$ не редуцируется относительно $S$. Предположим теперь, что $\operatorname{iv}(f) \cap O(\operatorname{lt} S) \neq \varnothing$, и покажем, что $f$ редуцируется к нормальной форме $\mathrm{NF}(f \downarrow S)$ относительно $S$.

Для каждого $\gamma \in \Gamma$ и каждого $\phi \in \mathcal{F}(a)$ вводится множество

$$
V_{\gamma}(\phi)=O(\operatorname{lt} S) \cap \operatorname{iv}(\phi) \cap U_{\gamma} .
$$

Это множество конечно или пусто, так как iv $(\phi)$ конечно. Определим элемент

$$
\gamma_{1}=\max \left\{\gamma \in \Gamma: V_{\gamma}(f) \neq \varnothing\right\}
$$

Пусть множество $V_{\gamma_{1}}(f)$ состоит из $m_{\gamma_{1}}$ элементов. Рассмотрим произвольный элемент $y \in V_{\gamma_{1}}(f)$. Согласно предположению найдется $g \in S$ такой, что $y \in O(\operatorname{lt} g)$. Следовательно, $f$ одношагово редуцируется к некоторому $r \in \mathcal{F}_{\gamma_{1}}(a)$ относительно $g$, причем $r$ не зависит от $y$ и $V_{\gamma_{1}}(r) \subset V_{\gamma_{1}}(g)$, поскольку $S$ - слабо разрешимое множество.

Если $V_{\gamma_{1}}(r) \neq \varnothing$, то выбираем произвольный элемент $\tilde{y} \in V_{\gamma_{1}}(r)$, ряд $\tilde{g} \in S$ такой, что $\tilde{y} \in O(\operatorname{lt} \tilde{g})$, и одношагово редуцируем $r$ относительно $\tilde{g}$ к некоторому $\tilde{r}$ такому, что $\tilde{y} \notin \operatorname{iv}(\tilde{r})$ и $V_{\gamma_{1}}(\tilde{r}) \subset V_{\gamma_{1}}(r)$. За $m_{\gamma_{1}}$ шагов мы редуцируем $f$ к некоторому $r_{m_{\gamma_{1}}}$ относительно $S$, и при этом множество $V_{\gamma_{1}}\left(r_{m_{\gamma_{1}}}\right)$ будет пустым.

Если найдется элемент $\gamma$ такой, что $V_{\gamma}\left(r_{m_{\gamma_{1}}}\right) \neq \varnothing$, то положим

$$
\gamma_{2}=\max \left\{\gamma \in \Gamma: V_{\gamma}\left(r_{m_{\gamma_{1}}}\right) \neq \varnothing\right\}
$$


Поступая описанным выше способом, редуцируем $r_{m_{\gamma_{1}}}$ к некоторому $r_{m_{\gamma_{2}}}$ относительно $S$, при этом $V_{\gamma_{2}}\left(r_{m_{\gamma_{2}}}\right)=\varnothing$. Продолжая этот процесс, получаем строго убывающую последовательность элементов $\gamma_{1}>\gamma_{2}>\cdots$. Эта последовательность не может быть бесконечной, так как любое подмножество во вполне упорядоченном множестве $Г$ имеет минимальный элемент. Следовательно, в этом случае $f$ редуцируется к нормальной форме $\mathrm{NF}(f \downarrow S)$.

Доказательство леммы 2 включает способ приведения сходящегося дифференциального ряда к нормальной форме. В общем случае нормальная форма ряда $f$ не единственна, однако для пассивных множеств справедливо следующее утверждение.

ПредЛОЖЕНИЕ 1. Пусть $S$ - слабо разрешимое пассивное множество в $\mathcal{F}(a)$ mакое, что lt $S=$ st $S$. Если $f \in \mathcal{F}(a) u \operatorname{iv}(f) \cap O($ lt $S) \neq \varnothing$, то $f$ редуиируется $\kappa$ единственной нормальной форме относительно $S$.

ДоказАтельство. Поскольку iv $(f) \cap O(\operatorname{lt} S) \neq \varnothing$, существование редукции к нормальной форме следует из леммы 2. Покажем единственность нормальной формы. Пусть $r_{1}, r_{2}$ - две различные нормальные формы ряда $f$ относительно $S$. Тогда разности $f-r_{1}, f-r_{2}$ и $r_{1}-r_{2}$ лежат в идеале $\left\langle\langle S\rangle\right.$. Согласно лемме 1 разность $r_{1}-r_{2}$ зависит хотя бы от одного элемента орбиты $O($ lt $S)$. С другой стороны, поскольку $r_{1}, r_{2}$ являются нормальными формами, они не зависят от элементов из орбиты $O($ lt $S)$. Следовательно, iv $\left(r_{1}-r_{2}\right) \cap O(\operatorname{lt} S)=\varnothing$, и мы приходим к противоречию.

Заметим, что подобное свойство единственности нормальной формы в случае кольца полиномов Бухбергер [10] использует в определении базиса Грёбнера.

Введем на полугруппе $\mathbb{N}^{n}$ бинарную операцию

$$
\alpha \diamond \beta=\left(\mu_{1}, \ldots, \mu_{n}\right), \quad \text { где } \quad \mu_{i}=\max \left(\alpha_{i}, \beta_{i}\right)-\alpha_{i} .
$$

ОПРЕДЕЛЕНиЕ 14. Пусть даны два сходящихся дифференциальных ряда из $\mathcal{F}(a)$ вида

$$
f_{1}=u_{\alpha}^{i}+h_{1}, \quad f_{2}=u_{\beta}^{i}+h_{2},
$$

где $u_{\alpha}^{i}=$ lt $f_{1}, u_{\beta}^{i}=$ lt $f_{2}$. Тогда разность

$$
D^{\alpha \diamond \beta} f_{1}-D^{\beta \diamond \alpha} f_{2}
$$

называется $\tau$-рядом om $f_{1}, f_{2}$ и обозначается как $\tau\left(f_{1}, f_{2}\right)$.

С помощью $\tau$-рядов получается критерий пассивности.

Лемма 3. Пусть $S$ - слабо разрешимое подмножество в $\mathcal{F}(a)$. Множество $S$ пассивно, а st $S=$ lt $S$ тогда и только тогда, когда для всех пар $f_{1}, f_{2} \in S$ ряд $\tau\left(f_{1}, f_{2}\right)$ редуцируется $\kappa$ нулю относителъно $S$.

ДокАЗАТЕЛЬство. Предположим, что $S$ - пассивное множество. Поскольку $f_{1}, f_{2} \in S$, то $\tau\left(f_{1}, f_{2}\right) \in\left\langle\langle S\rangle\right.$. Если ряд $\tau\left(f_{1}, f_{2}\right)$ не равен нулю, то согласно лемме 1 он зависит от элементов орбиты $O(\operatorname{lt} S)$. В соответствии с предложением 1 данный $\tau$-ряд редуцируется к нормальной форме относительно $S$. Эта нормальная форма не зависит от элементов орбиты $O(\operatorname{lt} S)$ и принадлежит идеалу $\langle\langle S\rangle$. Следовательно, она равна нулю. 
Пусть теперь для всех пар $f_{1}, f_{2} \in S$ ряд $\tau\left(f_{1}, f_{2}\right)$ редуцируется к нулю относительно $S$. Согласно соотношениям $(3.5)$, (3.6), (3.9) это означает, что существует конечная последовательность рядов $\left\{r_{i}\right\}_{i=1}^{p+1} \in \mathcal{F}(a)$, связанных равенствами

$$
r_{i}=q_{i} D^{\delta_{i}} g_{i}+r_{i+1}
$$

причем ряды $q_{i}, g_{i}, r_{i} \in \mathcal{F}(a)$ удовлетворяют условиям

$$
r_{0}=\tau\left(f_{1}, f_{2}\right), \quad r_{p+1}=0, \quad q_{i} \preceq r_{i}, \quad D^{\delta_{i}} g_{i} \preceq r_{i}, \quad r_{i+1} \preceq r_{i} .
$$

Таким образом, ряд $\tau\left(f_{1}, f_{2}\right)$ представляется в виде

$$
\tau\left(f_{1}, f_{2}\right)=\sum_{i=1}^{p} q_{i} D^{\delta_{i}} g_{i} .
$$

Легко видеть, что в силу (3.3) отношение $\preceq$ таково, что $h_{1} \preceq h$ и $h_{2} \preceq h$ влечет $h_{1} h_{2} \preceq h$, где $h_{1}, h_{2}, h \in \mathcal{F}(a)$. Тогда из формул (3.13), (3.14), (3.3) следует, что

$$
q_{i} D^{\delta_{i}} g_{i} \preceq r_{i}, \quad q_{i} D^{\delta_{i}} g_{i} \preceq \tau\left(f_{1}, f_{2}\right) .
$$

Следовательно, в соответствии с теоремой 1 работы [20] $S$ - пассивное множество.

ОПРЕДЕЛЕНИЕ 15. Пассивное множество $S \subset \mathcal{F}(a)$ называется каноническим, если каждый ряд $f \in S$ не редуцируется относительно $S \backslash f$.

Лемма 4. Пусть $S$ - слабо разрешимое пассивное множество в $\mathcal{F}(a)$ u lt $S=$ st $S$. Тогда существует единственное каноническое множество $R$ в $\mathcal{F}(a)$ такое, что $\langle\langle S\rangle\rangle=\langle\langle R\rangle\rangle$.

ДокАзАТЕЛЬство. Существование канонического множества доказывается непосредственным построением. Поскольку $S$ - конечное множество, найдутся элементы $\gamma_{1}<\gamma_{2}<\cdots<\gamma_{p}$ такие, что

$$
S=S_{\gamma_{1}} \cup S_{\gamma_{2}} \cup \cdots \cup S_{\gamma_{p}}
$$

где $S_{\gamma_{j}}=S \cap \Phi_{\gamma_{j}}$, а блоки $\Phi_{\gamma_{j}}$ задаются формулой (3.1). Рассмотрим множество $S_{\gamma_{1}}$. Поскольку $S$ - пассивное множество и $\gamma_{1}=\min \left\{\gamma \in \Gamma: S \cap \Phi_{\gamma} \neq \varnothing\right\}$, любой $f \in S_{\gamma_{1}}$ не зависит от элементов орбиты $O(\operatorname{lt}(S \backslash f))$.

Для каждого $j$ (где $2 \leqslant j \leqslant p$ ) введем два множества

$$
\begin{gathered}
Q_{j}=R_{\gamma_{1}} \cup \cdots \cup R_{\gamma_{j}}, \\
R_{\gamma_{j}}=\left\{\operatorname{NF}\left(f_{i} \downarrow Q_{j-1}\right) \neq \varnothing: f_{i} \in S_{\gamma_{j}}\right\} \cup\left\{f_{i} \in S_{\gamma_{j}}: f_{i} \nrightarrow Q_{j-1}\right\} .
\end{gathered}
$$

Здесь запись $f_{i} \nrightarrow Q_{j-1}$ означает, что $f_{i}$ не редуцируется относительно $Q_{j-1}$. Кроме того, мы полагаем $R_{\gamma_{1}}=S_{\gamma_{1}}$. Покажем, что $Q_{p}$ является каноническим множеством в $\mathcal{F}(a)$. Нужно показать, что любой $g \in Q_{p}$ не редуцируется относительно $Q_{p} \backslash g$.

Пусть ряд $g$ принадлежит $R_{\gamma_{j}}$. Очевидно, этот ряд не редуцируется относительно $R \backslash R_{\gamma_{j}}$. Заметим, что если ряд $r_{i}=\mathrm{NF}\left(f_{i} \downarrow Q_{j-1}\right)$, где $f_{i} \in S_{j}$, не равен нулю, то он принадлежит $\Phi_{\gamma_{j}}$. Действительно, если $r_{i} \in \Phi_{\gamma^{\prime}}\left(\right.$ где $\left.\gamma^{\prime}<\gamma_{j}\right)$, то iv $\left(r_{i}\right) \cap O(\operatorname{lt} S)=\varnothing$. $\mathrm{C}$ другой стороны, согласно лемме 1 любой элемент из $\langle\langle S\rangle\rangle$ зависит хотя бы от одного элемента из $O(\operatorname{lt} S)$. Мы приходим к противоречию. Значит, $R_{\gamma_{j}} \subset \Phi_{\gamma_{j}}$. 
Покажем, что если $g, h \in R_{\gamma_{j}}$ и lt $g=\mathrm{lt} h$, то $g=h$. Предположим, что это не так. Тогда $g-h \neq 0$ и $g-h \in \Phi_{\gamma^{\prime}}$, где $\gamma^{\prime}<\gamma_{j}$. Поскольку ряды $g, h$ не редуцируются относительно $Q_{j-1}$, их разность тоже не редуцируется относительно $Q_{j-1}$. По лемме 1 любой элемент из $\langle\langle S\rangle\rangle$ зависит хотя бы от одного элемента из $O(\operatorname{lt} S)$. Поэтому $g-h=0$, и существование доказано.

Остается доказать единственность. Предположим, что существует каноническое множество $\widetilde{R}$, не совпадающее с $R$. По условию $S$ является пассивным множеством и $\langle\langle R\rangle\rangle=\langle\langle\widetilde{R}\rangle\rangle=\langle\langle S\rangle$. Следовательно, верны равенства

$$
O(\operatorname{lt} S)=O(\operatorname{lt} R)=O(\operatorname{lt} \widetilde{R}), \quad \text { lt } R=\operatorname{lt} \widetilde{R} .
$$

Возьмем два элемента $g \in R, \tilde{g} \in \widetilde{R}$ таких, что lt $g=\operatorname{lt} \tilde{g}$. Покажем, что $g=\tilde{g}$. Предположим, что это неверно. Тогда $g-\tilde{g} \neq 0$ и $\mathrm{iv}(g-\tilde{g}) \cap O(\operatorname{lt} S) \neq \varnothing$ согласно лемме 1. Поскольку множества $R, \widetilde{R}$ канонические, ряды $g, \tilde{g}$ не зависят от элементов из орбит $O(\operatorname{lt}(R \backslash g))=O(\operatorname{lt}(\widetilde{R} \backslash \tilde{g}))$. Следовательно, разность $g-\tilde{g}$ не зависит от элементов орбиты $O(\operatorname{lt}(R \backslash g))$. Очевидно, верны формулы

$$
O(\operatorname{lt} S)=O(\operatorname{lt} R)=O(\operatorname{lt}(R \backslash g)) \cup O(\operatorname{lt} g), \quad \operatorname{iv}(g-\tilde{g}) \cap O(\operatorname{lt} g)=\varnothing .
$$

Таким образом, $\operatorname{iv}(g-\tilde{g}) \cap O(\operatorname{lt} S)=\varnothing$.

\section{4. ПОСТРОЕНИЕ ПАССИВНЫХ МНОЖКЕСТВ И ПРИМЕРЫ ДИФФЕРЕНЦИАЛЬНЫХ СИСТЕМ}

Если множество $S \subset \mathcal{F}(a)$ не является пассивным, то возникает проблема построения такого пассивного множества $P \subset \mathcal{F}(a)$, что $\langle\langle S\rangle\rangle=\langle\langle P\rangle\rangle$. Необходимо иметь удобный критерий существования пассивного множества для данного дифференциального идеала. Эти вопросы рассматривались в классических и современных работах, но, по-видимому, не нашли окончательного решения. Мы опишем схему построения пассивного множества, укажем на проблемы ее реализации и приведем некоторые примеры.

Сначала докажем утверждение, которое можно использовать при исследовании пассивности. Обозначим через $\mathbb{K}_{n}(c)$ алгебру сходящихся степенных рядов (с центром в точке $\left.c \in \mathbb{K}^{n}\right)$, зависящих от $n$ переменных $x_{1}, \ldots, x_{n}$. Множество

$$
\mathfrak{m}_{n}(c)=\left\{f \in \mathbb{K}_{n}(c): f(c)=0\right\}
$$

образует максимальный идеал алгебры $\mathbb{K}_{n}(c)$. Нам понадобится следующее уточнение теоремы о неявных функциях.

ПрЕДЛОЖЕНИЕ 2. Пустъ $f_{1}, \ldots, f_{k} \in \mathfrak{m}_{n}(c)$ и якобиан $\frac{\partial\left(f_{1}, \ldots, f_{k}\right)}{\partial\left(x_{1}, \ldots, x_{k}\right)}(c)$ не равен нулю. Тогда существуют рядь $g_{1}, \ldots, g_{k} \in \mathfrak{m}_{n}(c)$ вида

$$
g_{i}=x_{i}+h_{i}
$$

и квадратная матрица $A$-го порядка с элементами из $\mathbb{K}_{n}(c)$ такая, что

$$
\left(f_{1}, \ldots, f_{k}\right)=\left(g_{1}, \ldots, g_{k}\right) A, \quad A(c)=\left(\frac{\partial f_{j}}{\partial x_{l}}\right)(c),
$$

где $j, l \in \mathbb{N}_{k}$, и при этом рядъ $h_{i}$ не зависят от $x_{1}, \ldots, x_{k}$. 
ДокАЗАТЕЛЬСтво. Из условий предложения и теоремы о неявных функциях следует, что существуют ряды $g_{1}, \ldots, g_{k} \in \mathfrak{m}_{n}(c)$ вида (4.1) такие, что

$$
f_{i}\left(-h_{1}, \ldots,-h_{k}, x_{k+1}, \ldots, x_{n}\right)=0, \quad i \in \mathbb{N}_{k} .
$$

Из теоремы Вейерштрасса о делении [18] вытекает представление [20]

$$
f_{j}=\sum_{i=1}^{k} g_{i} a_{j i}+r_{j}, \quad j \in \mathbb{N}_{k},
$$

где $r_{j}, a_{i, j} \in \mathbb{K}_{n}(c)$ и $\operatorname{iv}\left(r_{j}\right) \cap\left\{x_{1}, \ldots, x_{k}\right\}=\varnothing$. Подставим в (4.4) вместо $x_{1}, \ldots, x_{k}$ ряды $-h_{1}, \ldots,-h_{k}$. Тогда из соотношений (4.1), (4.3) следует, что ряды $r_{j}$ нулевые. Следовательно, первая формула в (4.2) верна.

Докажем, что верна вторая формула в (4.2). Дифференцируя равенство (4.4) по $x_{l}$ (при $r=0, l \in \mathbb{N}_{k}$ ), получим

$$
\frac{\partial f_{j}}{\partial x_{l}}=\sum_{i=1}^{k} \frac{\partial g_{i}}{\partial x_{l}} a_{j i}+\sum_{i=1}^{k} g_{i} \frac{\partial a_{j i}}{\partial x_{l}}
$$

Поскольку для всех $i, l \in \mathbb{N}_{k}$ выполняются равенства

$$
g_{i}(c)=0, \quad \frac{\partial g_{i}}{\partial x_{l}}(c)=\delta_{l}^{i}
$$

где $\delta_{l}^{i}$ - символ Кронекера, из (4.5) следует, что $\frac{\partial f_{j}}{\partial x_{l}}(c)=a_{j l}(c)$. Это доказывает вторую формулу в (4.2).

Вернемся к изучению свойств алгебры $\mathcal{F}(a)$.

ОПРЕДЕЛЕНИЕ 16. Будем говорить, что кортежи $\vec{f}^{1}, \vec{f}^{2} \in \mathcal{F}^{k}(a)$ подобны, если существует невырожденная в точке $a$ квадратная матрица $A k$-го порядка с элементами из $\mathcal{F}(a)$ такая, что $\vec{f}^{1}=\vec{f}^{2} A$. Два множества $R_{1}, R_{2} \subset \mathcal{F}(a)$ из $k$ элементов называются эквивалентными, если кортежи $\vec{f}^{1}, \vec{f}^{2} \in \mathcal{F}^{k}(a)$, составленные из элементов множеств $R_{1}, R_{2}$ соответственно, являются подобными.

Очевидно, эквивалентные множества порождают совпадающие дифференциальные идеалы. Используя эквивалентные множества, можно в определенных случаях решить проблему построения пассивного множества для данного дифференциального идеала, порожденного конечным подмножеством в $\mathcal{F}(a)$. Приведем схему построения пассивного множества.

Пусть $\widehat{\mathcal{F}}$ является стратифицированным $\mathbb{N}_{-0}^{n}$-множеством. Предположим, что $S=\left\{f_{1}, \ldots, f_{k}\right\}$ не является слабо разрешимым множеством в $\mathcal{F}$ и существует точка $c$, в которой обращаются в ноль все ряды из $S$, а ранг матрицы Якоби $\left(\frac{\partial f_{j}}{\partial z_{i}}\right)(c)$ равен $p>0$ (здесь $z_{i}$ - переменные, от которых зависят ряды $f_{j} \in S$ ). Тогда найдется минор $\frac{\partial\left(f_{i_{1}}, \ldots, f_{i_{p}}\right)}{\partial\left(z_{i_{1}}, \ldots, z_{i_{p}}\right)}(c)$, не равный нулю. Согласно предложению 2 существуют ряды вида

$$
g_{1}=z_{i_{1}}+h_{1}, \quad \ldots, \quad g_{p}=z_{i_{p}}+h_{p}
$$

такие, что кортежи $\left(f_{i_{1}}, \ldots, f_{i_{p}}\right), g_{1} \ldots, g_{p}$ подобны. Если $h_{1} \prec z_{i_{1}}, \ldots, h_{p} \prec z_{i_{p}}$, то множество $\widetilde{S}=\left\{g_{1}, \ldots, g_{p}\right\}$ слабо разрешимо. Проверяем, что любой ряд $f \in S$ 
редуцируется к нулю относительно $\widetilde{S}$. Затем редуцируем все $\tau$-ряды вида $(3.11)$, составленные из всех пар $f_{1}, f_{2} \in \widetilde{S}$, к нормальным формам относительно $\widetilde{S}$. Обозначим через $S_{1}$ множество нормальных форм, не равных нулю. Если множество $S_{1}$ не является слабо разрешимым, то к нему применяем описанную выше процедуру и получаем слабо разрешимое множество $\widetilde{S}_{1}$. Далее проверяем множество $\widetilde{S} \cup \widetilde{S}_{1}$ на пассивность, редуцируя $\tau$-ряды к нормальным формам относительно $\widetilde{S} \cup \widetilde{S}_{1}$. Если $\widetilde{S} \cup \widetilde{S}_{1}$ не пассивно, то, поступая описанным выше способом, получаем множество $\widetilde{S}_{2}$ и множество $\widetilde{S} \cup \widetilde{S}_{1} \cup \widetilde{S}_{2}$. Проверяем последнее множество на пассивность и так далее. Как будет показано ниже, после конечного числа шагов мы получим пассивное множество.

Чтобы доказать конечность числа шагов в описанной выше схеме, рассмотрим кольцо $\mathcal{M}$ многочленов $\mathbb{K}\left[x_{1}, \ldots, x_{n}\right]$ и векторное пространство $\mathbb{K} U$ над $\mathbb{K}$, порожденное множеством $U(2.2)$. Несложно доказать [20], что $\mathbb{K} U$ можно наделить структурой левого $\mathcal{M}$-модуля, и этот модуль изоморфен нётерову модулю $\mathcal{M}^{n}$. Обозначим объединение $\widetilde{S} \cup \widetilde{S}_{1} \cdots \cup \widetilde{S}_{i}$ через $Q_{i}$, а $\widetilde{S}$ - через $Q_{0}$. Предположим, что для любого $i$ множество $Q_{i}$ строго содержится в $Q_{i+1}$. Этой строго возрастающей цепочке множеств соответствует строго возрастающая цепочка старших членов

$$
\text { lt } Q_{0} \subset \operatorname{lt} Q_{1} \subset \cdots \subset \operatorname{lt} Q_{i} \subset \cdots \text {. }
$$

Включения в этой цепочке строгие, так как $\widetilde{S}_{i}$ состоит из нормальных форм относительно $Q_{i-1}$. В свою очередь, последней цепочке соответствует цепочка подмодулей

$$
\left[\operatorname{lt} Q_{0}\right] \subset\left[\operatorname{lt} Q_{1}\right] \subset \cdots \subset\left[\operatorname{lt} Q_{i}\right] \subset \cdots,
$$

где $\left[\right.$ lt $\left.Q_{i}\right]$ - подмодуль $\mathcal{M}$-модуля $\mathbb{K} U$, порожденный множеством $Q_{i}$. Поскольку модуль $\mathbb{K} U$ нётеров, у него не может существовать строго возрастающей бесконечной цепочки подмодулей. Мы пришли к противоречию.

В общем случае мы не можем гарантировать, что описанная выше схема работает, поскольку могут не выполняться условия предложения 2.

Приведем примеры построения пассивных множеств.

Пример 1. Пусть $\mathbb{K}=\mathbb{R}$ - поле вещественных чисел, $m=1, n=2$. Рассмотрим множество $S=\left\{f_{1}, f_{2}\right\} \subset \mathcal{F}$, состоящее из двух сходящихся дифференциальных рядов

$$
f_{1}=u_{(1,1)}-\sin u_{(0,0)}, \quad f_{2}=u_{(0,3)}+\frac{1}{2} u_{(0,1)}^{3} .
$$

Множество $U$ разбивается на подмножества $U_{i}=\left\{u_{\alpha}:|\alpha|=i\right\}$ и становится стратифицированным $\mathbb{N}_{-0}^{2}$-множеством. Мы хотим найти пассивное множество $Q \subset \mathcal{F}(a)$ такое, что $\langle\langle Q\rangle\rangle=\left\langle\langle S\rangle\right.$. Ограничения на точку $a \in \mathbb{K}^{n+\infty}$ возникают в процессе вычислений.

Далее будут использоваться более привычные обозначения

$$
D_{t}=D_{1}, \quad D_{x}=D_{2}, \quad u=u_{(0,0)}, \quad u_{t}=u_{(1,0)}, \quad u_{x}=u_{(0,1)}, \quad u_{t x}=u_{(1,1)} .
$$

Множеству $S$ соответствует система уравнений с частными производными

$$
u_{t x}-\sin u=0, \quad u_{x x x}+\frac{1}{2} u_{x}^{3}=0 .
$$


Вычисляем $\tau$-ряд от $f_{1}, f_{2}$ :

$$
\tau\left(f_{1}, f_{2}\right)=D_{x}^{2} f_{1}-D_{t} f_{2}=u_{x}^{2} \sin u-u_{x x} \cos u-\frac{3}{2} u_{t x} u_{x}^{2} .
$$

Очевидно, ряд $\tau\left(f_{1}, f_{2}\right)$ редуцируется к нормальной форме

$$
f_{3}=-u_{x x} \cos u-\frac{1}{2} u_{x}^{2} \sin u
$$

относительно $S$. Следовательно, согласно лемме 1 множество $S$ не является пассивным. Если $\cos u \neq 0$, то $f_{3}$ эквивалентен ряду $\tilde{f}_{3}=u_{x x}+u_{x}^{2} \operatorname{tg}(u) / 2$.

Теперь нужно проверить на пассивность множество $S_{1}=\left\{f_{1}, f_{2}, \tilde{f}_{3}\right\}$. Вычисляя $\tau$-ряд

$$
\tau\left(f_{1}, \tilde{f}_{3}\right)=D_{x}\left(u_{t x}-\sin u\right)-D_{t}\left(u_{x x}+\frac{u_{x}^{2}}{2} \operatorname{tg} u\right)=-u_{x} \cos u-u_{t x} u_{x} \operatorname{tg} u-\frac{u_{t} u_{x}^{2}}{2 \cos ^{2} u}
$$

и редуцируя его к нормальной форме относительно $S_{1}$, получаем

$$
f_{4}=-\frac{u_{x}}{2 \cos ^{2} u}\left(u_{t} u_{x}+2 \cos u\right) .
$$

Если $u_{x} \neq 0$, то $f_{4}$ эквивалентен ряду

$$
\tilde{f}_{4}=u_{t}+\frac{2 \cos u}{u_{x}}
$$

Несложно вычислить, что ряды $f_{1}, f_{2}$ лежат в идеале $\left\langle\left\langle f_{3}, f_{4}\right\rangle\right\rangle$ и ряд $\tau\left(\tilde{f}_{3}, \tilde{f}_{4}\right)$ редуцируется к нулю относительно множества $\left\{\tilde{f}_{3}, \tilde{f}_{4}\right\}$.

Таким образом, $\left\{\tilde{f}_{3}, \tilde{f}_{4}\right\}$ является искомым пассивным множеством. Это пассивное множество порождает тот же дифференциальный идеал, что и первоначальное множество $\left\{f_{1}, f_{2}\right\}$. Его можно использовать, чтобы найти решения системы (4.6). Для этого достаточно проинтегрировать систему

$$
\tilde{f}_{3}=0, \quad \tilde{f}_{4}=0 .
$$

Сначала рассмотрим первое уравнение

$$
u_{x x}+\frac{u_{x}^{2}}{2} \operatorname{tg} u=0
$$

системы (4.7). Разделим данное уравнение на $u_{x}$ и найдем первый интеграл

$$
\frac{u_{x}}{\sqrt{\cos u}}=c
$$

где $c$ - пока произвольная функция от $t$. Подставляя первый интеграл во второе уравнение системы (4.7), получим систему первого порядка

$$
u_{x}-c \sqrt{\cos u}=0, \quad u_{t}+\frac{2 \sqrt{\cos u}}{c}=0 .
$$


Составляя $\tau$-ряд от левых частей системы (4.8) и редуцируя его к нормальной форме, видим, что нормальная форма равна нулю только при $c=$ const. В этом случае неявное решение системы (4.8) имеет вид

$$
\int \frac{d u}{\sqrt{\cos u}}=c x-\frac{2 t}{c}+c_{1},
$$

где $c_{1}$ - произвольная константа. Полученное решение уравнения синус-гордон инвариантно относительно преобразований растяжений и переносов.

ПримеР 2. В качестве второго примера рассмотрим уравнение акустики в неоднородной среде [22]

$$
\frac{p_{t t}}{\rho c^{2}}=\left(\frac{p_{x}}{\rho}\right)_{x}+\left(\frac{p_{y}}{\rho}\right)_{y}+\left(\frac{p_{z}}{\rho}\right)_{z},
$$

где $p$ - давление, $\rho$ - плотность, $c$ - скорость звука. Давление является искомой функцией, а плотность и скорость звука - заданными функциями от $x, y, z$. Будем считать сначала, что $\rho$ и $c$ - произвольные положительные функции. Если ввести новую функцию $P=p / \sqrt{\rho}$, то уравнение (4.9) принимает вид

$$
P_{t t}=c^{2}(\Delta P+q P) .
$$

Здесь $\Delta$ - трехмерный оператор Лапласа, а функция $q$ выражается через $\rho$ :

$$
q=-\frac{\Delta(\sqrt{\rho})}{\sqrt{\rho}}
$$

Мы хотим найти некоторые решения уравнения (4.10). Предположим, что $P$ удовлетворяет дополнительному уравнению

$$
P_{t}=a P_{x}+b P_{y}
$$

считая, что $a, b, c$ являются функциями только переменной $x$.

Уравнениям (4.10), (4.11) соответствуют два дифференциальных ряда

$$
e_{0}=P_{t t}-c^{2}(\Delta P+q P), \quad e_{1}=P_{t}-a P_{x}-b P_{y} .
$$

Множество $S=\left\{e_{0}, e_{1}\right\}$ не является пассивным при произвольных функциях $a, b, c$. Получим условия, при выполнении которых множество $S$ становится пассивным. Для этого редуцирируем $e_{0}$ относительно $e_{1}$ к нормальной форме, т. е. избавимся от слагаемого $P_{t t}$ в $e_{0}$. Несложно видеть, что нормальная форма имеет вид

$$
\begin{aligned}
e_{2} & =e_{0}-D_{t} e_{1}-a D_{x} e_{1}-b e_{1}= \\
& =\left(a^{2}-c^{2}\right) P_{x x}+a\left(a^{\prime}+2 b\right) P_{x}-c^{2}\left(P_{y y}+P_{z z}\right)+\left(a b^{\prime}+b^{2}-q c^{2}\right) P .
\end{aligned}
$$

Предположим теперь, что $c=a, b=-a^{\prime} / 2$. Тогда $e_{2}$ заметно упрощается:

$$
e_{2}=-a^{2}\left(P_{y y}+P_{z z}\right)+\left(-\frac{a a^{\prime \prime}}{2}+\frac{\left(a^{\prime}\right)^{2}}{4}-q a^{2}\right) P .
$$


При $a \neq 0$ ряд $e_{2}$ эквивалентен

$$
\tilde{e}_{3}=P_{y y}+P_{z z}+\left(\frac{a a^{\prime \prime}}{2 a}+\frac{\left(a^{\prime}\right)^{2}}{4 a^{2}}-q\right) P .
$$

Предположим теперь, что

$$
q=-\frac{a a^{\prime \prime}}{2 a}+\frac{\left(a^{\prime}\right)^{2}}{4 a^{2}}+h,
$$

где $h$ зависит только от $y$ и $z$. Тогда ряд $\tilde{e}_{3}$ будет иметь вид

$$
\tilde{e}_{3}=P_{y y}+P_{z z}+h P \text {. }
$$

Несложно видеть, что множество $S_{1}=\left\{e_{1}, \tilde{e}_{3}\right\}$ пассивное и

$$
e_{0}=D_{t} e_{1}+a D_{x} e_{1}+b e_{1}-a^{2} \tilde{e}_{3}
$$

Следовательно, некоторые решения уравнения (4.10) можно найти, решив систему

$$
e_{1}=0, \quad \tilde{e}_{3}=0 .
$$

Решение первого уравнения

$$
P_{t}-a P_{x}+a^{\prime} \frac{P}{2}=0
$$

системы (4.12) сводится к интегрированию обыкновенных дифференциальных уравнений

$$
\frac{d t}{1}=\frac{d x}{a}=-2 \frac{d P}{a^{\prime} P}
$$

Следовательно, общее решение уравнения (4.13) имеет вид

$$
P=\sqrt{a(x)} f\left(t-\int \frac{d x}{a(x)}, y, z\right) .
$$

Согласно второму уравнению системы (4.12) функция $f$ должна удовлетворять уравнению

$$
f_{y y}+f_{z z}+h f=0 .
$$

Если $h=0$, то получаем уравнение Лапласа. При специальном выборе функции $h$ уравнение (4.14) сводится к уравнению Лапласа [23]. Если функции $f$ и $h$ не зависят от $z$, то (4.14) становится линейным обыкновенным уравнением

$$
f^{\prime \prime}+h f=0
$$

которое при специальном выборе $h$ может быть проинтегрировано в явном виде [24].

Благодарности. Автор благодарен рецензенту за полезные замечания, позволившие улучшить изложение результатов статьи. Работа выполнена при финансовой поддержке РФФИ (гранты № 12-01-00648_a, 10-01-00435_а), а также при поддержке гранта Правительства РФ для проведения исследований под руководством ведущих ученых в Сибирском федеральном университете (договор № 14.Ү26.31.0006). 


\section{Список литературы}

[1] J. Molk (ed.), Encyclopédie des sciences mathématiques. II, v. 4: Équations aux dérivées partielles, Jacques Gabay, Paris, 1916.

[2] M. E. Goursat, Leçons sur l'intégration des équations aux dérivées partielles du second order à deux variables indépendantes, v. II, Librairie scientifique A. Hermann, Paris, 1898.

[3] M. Kuranishi, Lectures on Involutive Systems of Partial Differential Equations, Publ. Soc. Mat., São Paulo, 1967.

[4] Д. Спенсер, "Переопеределенные системы линейных дифференциальных уравнений в частных производных", Сб. переводов, Математика, 14, вып. 3, Мир, М., 1970, 99-126.

[5] Ж.-Ф. Поммаре, Системы уравнений с частными производными и псевдогруппь Ли, Мир, М., 1983.

[6] А.М. Виноградов, "Геометрия нелинейных дифференциальных уравнений", Итоги науки и техн. Сер. Пробл. геом., 11, ВИНИТИ, М., 1980, 89-134.

[7] A. D. Wittkopf, Algorithms and implementations for differential elimination, PhD. Thesis, Simon Fraser University, Burnaby, British Columbia, Canada, 2004.

[8] M. Marvan, Found. Comput. Math., 9:6 (2009), 651-674.

[9] V.P. Gerdt, Yu. A. Blinkov, "Janet-like monomial division", Computer Algebra in Scientific Computing/CASC 2005 (Kalamata, Greece, September 12-16, 2005), Lecture Notes in Computer Science, 3718, Springer, Berlin, 2005, 174-183; "Janet-like Gröbner bases", Computer Algebra in Scientific Computing/CASC 2005 (Kalamata, Greece, September 12-16, 2005), Lecture Notes in Computer Science, 3718, Springer, Berlin, 2005, 184-195.

[10] Б. Бухбергер, "Базисы Грёбнера. Алгоритмический метод в теории полиномиальных идеалов", Компьютерная алгебра. Символьные и алгебраические вычисления, ред. Б. Бухбергер, Дж. Коллинз, Р. Лоос, Мир, М., 1986, 331-372.

[11] Д. Кокс, Дж. Литтл, Д. О’Ши, Идеаль, многообразия и алгоритмы, Мир, М., 2000.

[12] Л. В. Овсянников, Групповой анализ дифференииалъных уравнений, Наука, М., 1978.

[13] Н. Х. Ибрагимов, Группь преобразований в математической физике, Наука, М., 1983.

[14] А.Ф. Сидоров, В.П. Шапеев, Н.Н. Яненко, Метод дифференииальных связей и его приложения в газовой динамике, Наука, Новосибирск, 1984.

[15] В. И. Фущич, А. Г. Никитин, Симметрия уравнений квантовой механики, Наука, М., 1990.

[16] М. Абловиц, Х. Сигур, Солитоны и метод обратной задачи, Мир, М., 1987.

[17] М. Атья, И. Макдональд, Введение в коммутативную алгебру, Мир, М., 1972.

[18] Г. Грауэрт, Р. Реммерт, Аналитические локальные алгебры, Наука, М., 1988.

[19] J. Ritt, Differential Algebra, AMS, New York, 1950.

[20] О. В. Капцов, Программирование, 40:2 (2014), 32-40.

[21] О. Зарисский, П. Самюэль, Коммутативная алгебра, т. 2, ИЛ, М., 1963.

[22] Л. М. Бреховских, Волны в слоистых средах, Наука, М., 1973.

[23] О.В. Капцов, Методы интегрирования уравнений с частными производными, Физматлит, М., 2009.

[24] V.B. Matveev, M. A. Salle, Darboux Trasformations and Solitons, Springer, New York, 1991. 